\title{
Philosophiques
}

\section{Panaccio, Claude, Le discours intérieur. De Platon à Guillaume d'Ockham, Paris, Éditions du Seuil, 1999, 341 pages.}

\section{Guy Hamelin}

Volume 29, numéro 1, printemps 2002

Spinoza sous le prisme de son anthropologie

URI : https://id.erudit.org/iderudit/009570ar

DOI : https://doi.org/10.7202/009570ar

Aller au sommaire du numéro

Éditeur(s)

Société de philosophie du Québec

ISSN

0316-2923 (imprimé)

1492-1391 (numérique)

Découvrir la revue

Citer ce compte rendu

Hamelin, G. (2002). Compte rendu de [Panaccio, Claude, Le discours intérieur. De Platon à Guillaume d'Ockham, Paris, Éditions du Seuil, 1999, 341 pages.]

Philosophiques, 29(1), 147-150. https://doi.org/10.7202/009570ar d'utilisation que vous pouvez consulter en ligne.

https://apropos.erudit.org/fr/usagers/politique-dutilisation/ 


\title{
Comptes rendus
}

\author{
Panaccio, Claude, Le discours intérieur. De Platon à Guillaume \\ d'Ockham, Paris, Éditions du Seuil, 1999, 341 pages.
}

" A single word even may be a spark of inextinguishable thought ». Exprimée par le poète britannique Percy B. Shelley, cette réflexion illustre bien la motivation parfois simple d'entreprises souvent colossales. C'est le cas de la plus récente étude produite par Claude Panaccio. Ce dernier part, en effet, d'une notion élémentaire, le logos, et ses nombreux équivalents latins, comme le uerbum, pour en arriver à une exposition détaillée de son évolution à travers l'histoire. Concept clé en philosophie, le logos a besoin, de fait, d'un traitement particulier qui outrepasse tout cadre étriqué. Comme l'indique le titre de l'ouvrage, l'examen porte avant tout sur le caractère intérieur de ce discours. L'oratio in mente, selon l'expression utilisée, entre autres, par Boèce et Guillaume d'Ockham, est ainsi décrite par l'auteur : « [...] il existe dans les esprits individuels [...] des représentations mentales qui, bien qu'indépendantes des langues de communication, sont combinables entre elles en unités plus complexes, de la même façon précisément que les mots de la langue se combinent en phrases. ». (p. 17) Dans son élaboration la plus articulée, comme chez Ockham, il s'agit d'une théorie qui met l'accent à la fois sur le caractère universel et naturel de ce discours intérieur, dans la mesure où celui-ci est partagé par tous les individus et est constitué de concepts considérés comme des signes naturels des choses extérieures. En outre, cette parole intérieure comporte des catégories syntaxiques (nom, verbe, etc.) et des fonctions sémantiques (significatio, connotatio, suppositio) comparables à celles des langages conventionnels, ce qui implique l'existence d'une pensée logiquement organisée et dotée d'une structure compositionnelle. Quant à la valeur de vérité des jugements mentaux, elle est fonction de la référence (suppositio) de ses parties constituantes, à savoir les concepts qui tiennent lieu de sujet ou de prédicat. Prenant pour point de départ ce traitement ockhamiste passablement exhaustif, Panaccio relate, dans une enquête historique minutieuse et circonstanciée, l'origine de cette idée de langage mental à partir des premiers éléments rencontrés chez les chefs de file de l'Antiquité grecque et en suit les principales filières de transmission dans les écoles de pensée les plus diverses jusqu'à son épanouissement au XIV ${ }^{\mathrm{e}}$ siècle. L'A. signale, dès les premières lignes de l'avant-propos, que le projet de son étude était, à l'origine, plus restreint et modeste : «[...] il s'agissait de retracer les discussions théoriques des années 12501320 environ qui conduisirent à la systématisation par Guillaume d'Ockham de l'idée de discours mental (oratio mentalis) ». (p. 13) Les transformations majeures apportées par la suite, notamment en ce qui a trait à l'extension de la période examinée, n'ont pas nui au résultat obtenu, car l'ensemble du travail conserve la marque d'une qualité exceptionnelle qui a, d'ailleurs, été soulignée par l'Académie des Sciences morales et politiques de l'Institut de France avec l'attribution, une année seulement après la parution de l'ouvrage, du prix Grammaticakis-Neumann. L'élargissement du cadre initial est donc, sans aucun doute, le résultat d'une pensée inextinguible.

Dès les premières pages du livre, l'A. explique l'origine de l'intérêt pour cette question du discours intérieur. Ce point est particulièrement éclairant pour comprendre l'ensemble de sa démarche intellectuelle. La motivation est double. D'abord, la problématique contemporaine du language of thought, telle que développée notam- 
ment par le philosophe américain Jerry A. Fodor, a joué, dès le départ, un rôle déterminant. La ressemblance entre cette dernière question et le thème du discours intérieur chez Ockham fut considérée par l'A. suffisamment importante pour établir certaines corrélations précises. Cette première raison peut surprendre l'historien de la pensée confiné à son seul objet d'étude, mais n'étonnera pas le lecteur déjà familiarisé avec l'œuvre de Panaccio, notamment avec son précédent livre intitulé Les mots, les concepts et les choses. La sémantique de Guillaume d'Occam et le nominalisme d'aujourd'hui (Paris/Montréal, Vrin/Bellarmin, 1991, 288 p.), dans lequel il met en parallèle, comme l'indique le titre, les idées d'Ockham au sujet du nominalisme avec celles d'auteurs contemporains comme Fodor, Davidson, Goodman et bien d'autres. L'A. défend lui-même résolument le point de vue selon lequel il existe des thèmes philosophiques de nature, en quelque sorte, éternelle, dans la mesure où ceux-ci sont de tous les temps et transcendent, du moins partiellement, les contextes historiques. Certaines données théoriques sont "transtemporelles ", affirme Panaccio, qui ne cache pas son étonnement devant les nombreux recoupements rencontrés dans des doctrines développées sur un même sujet à plus de cinq siècles d'intervalle. En conséquence, il n'existe apparemment aucune raison valable de douter que l'examen détaillé des principaux questionnements proposés, à une certaine époque, par une théorie sur un thème précis puisse, de fait, nous aider à mieux comprendre et à résoudre certains problèmes rencontrés, à une autre époque, dans d'autres doctrines sur un sujet analogue, comme c'est le cas avec la question du discours intérieur. Bien qu'il s'agisse de l'une des deux principales raisons pour lesquelles Panaccio s'est, au départ, intéressé au problème du langage intérieur, la présente étude n'établit aucune comparaison avec la question contemporaine du language of thought, à l'exception d'un court développement qui se trouve dans la conclusion. La seconde motivation de l'A. est exprimée dans l'hypothèse initiale de l'ouvrage : « [...] Guillaume d'Ockham accomplit, dans les années 1315-1325, une révolution théorique majeure et extrêmement influente, par la mise au point précisément de ce concept d'oratio mentalis ». (p. 14) Cette conjecture originelle, qui sous-tend l'ensemble de l'étude, se verra confirmée par la suite. L'objectif de l'A. consiste, dans ce contexte, à retracer la source historique de cette idée de logos intérieur et à en suivre la formation, afin de faire ressortir le caractère innovateur, systématique et achevé de l'entreprise ockhamiste. Présente dès le début du projet, cette double approche rétrospective, qui se réduit d'abord à examiner la position d'Ockham à partir de l'intérêt suscité par la problématique contemporaine et, ensuite, à remonter aux origines de la philosophie occidentale à partir de la conception du Venerabilis inceptor, permet donc de faire l'histoire d'un thème en identifiant, avant tout, comme le signale Panaccio : «[...] les problèmes que les auteurs entendaient traiter à l'aide d'une telle notion (discours intérieur) et de décrire les rôles précis qu'ils lui confiaient dans leurs discussions théoriques ». (p. 22)

L'ouvrage permet avant tout de découvrir la grande variété des débats théoriques dans lesquels est apparue la problématique du langage intérieur, ainsi que la richesse des nombreuses interprétations qu'elle a suscitée. Il nous est cependant impossible d'illustrer, dans le présent contexte, toute la valeur des thèmes abordés, ainsi que toute la diversité des auteurs examinés dans cette étude. Pour l'essentiel, précisons que l'exposé se divise en trois grandes sections. La partie initiale retrace d'abord les premiers éléments relatifs au discours intérieur rencontrés chez les Grecs anciens. Platon discute déjà du caractère dialogique de la pensée, alors qu'Aristote met plutôt l'accent sur sa nature logique, laissant ainsi présupposer une certaine com- 
positionnalité. Il n'en demeure pas moins que l'idée du discours intérieur est, chez ces deux protagonistes, assez peu développée et ne fait l'objet d'aucun traitement spécifique de nature syntaxique ou sémantique. Par la suite, le thème du logos endiathetos (discours intérieur) s'impose à la presque totalité de la communauté philosophique, bien qu'il soit parfois interprété différemment par ses principaux défenseurs. Dans la plupart des cas, l'expression s'oppose à celle de logos prophorikos, c'est-à-dire le discours proféré par la voix. Au deuxième siècle de notre ère, une nouvelle tradition doctrinale, cette fois-ci d'origine chrétienne, surgit dans les milieux intellectuels et prend un essor considérable qui atteindra son apogée, quelques siècles plus tard, avec Augustin. Cette dernière conception renouvelle profondément l'idée de langage intérieur à partir de préoccupations théologiques, encore qu'elle incorpore certains éléments de l'héritage grec. La section se termine par l'examen des positions défendues sur le thème du discours intérieur par certains néo-platoniciens, comme Ammonius et Porphyre, et par Boèce, le principal propagateur de la conception grecque chez les Latins, sans compter un survol réservé à la contribution des principaux penseurs arabes. La question fondamentale de savoir si le langage mental est ou non universel, chez ces néo-platoniciens, retient principalement l'attention. Il s'agit de vérifier dans ce cas-ci, comme dans bien d'autres qui apparaîtront par la suite, si le discours intérieur est lié à une langue particulière, comme le latin, ou s'il est complètement indépendant de toute langue de communication, à l'exemple du uerbum in corde chez Augustin. La deuxième partie de l'ouvrage fait le point au sujet de la réception, dans l'Université du XIII ${ }^{\mathrm{e}}$ siècle, de ces deux principales traditions anciennes, la grecque et la chrétienne, à l'égard du discours mental. La section se subdivise en quatre parties distinctes. L'A. retrace d'abord les principales doctrines qui ont perpétué le thème en question du XI ${ }^{\mathrm{e}}$ siècle jusqu'à Ockham. Parmi les penseurs étudiés, Anselme présente une synthèse partielle des deux grands héritages du passé sur le discours intérieur, "en identifiant le verbe mental d'Augustin aux similitudines du Perihermeneias.". (p. 158) Panaccio examine, dans les chapitres subséquents, certaines questions précises qui ont contribué à sophistiquer la problématique de l'oratio mentalis, comme celle du statut ontologique du verbe mental ou celle du concept considéré en tant que signe ou encore celle de l'objet de la logique. La position de Thomas d'Aquin constitue une phase importante de l'évolution du concept de discours intérieur avec l'ajout d'une étape à la théorie aristotélicienne de la connaissance. Selon l'Aquinate, l'intellect possible produit un pur objet de pensée identifié au langage mental. Par delà les catégories aristotéliciennes, ce mode d'être particulier sera repris, dans un premier temps, par Ockham dans sa théorie dite du fictum. Entraînant plus d'inconvénients que de bénéfices, cet objet purement intentionnel sera, par la suite, éliminé par le Venerabilis inceptor, qui prendra notamment en considération les arguments du fameux principe connu sous le nom de rasoir d'Ockham. La troisième et dernière partie du livre est consacrée à la doctrine de l'oratio mentalis chez Guillaume d'Ockham et aux principales réactions qu'elle a suscitées dans les universités d'Angleterre et de France. On constate surtout, dans cette section, que rien de comparable à la position défendue par Ockham sur le langage mental n'avait été proposé avant lui. L'A. nous entraîne, enfin, au cœur des intenses discussions qui sont survenues au sujet de cette théorie ockhamiste chez les dominicains et les franciscains, ainsi que chez les Maîtres de la Faculté des arts.

L'idée de discours intérieur a toujours intrigué les penseurs. Les attributs accomplis d'un tel langage, comme l'absence d'ambiguïté sémantique ou le caractère 
universel et commun à tous, en font rêver plusieurs qui aimeraient bien les voir transposés aux diverses langues de communication. Rousseau n'exprime-t-il pas, comme bien d'autres d'ailleurs, son souhait en affirmant : "s'il y avait une langue naturelle et commune à tous les hommes ». Bien que cette dernière aspiration semble bien irréalisable, l'hypothèse d'un discours intérieur a, par contre, été prise au sérieux par certains philosophes au cours de l'histoire. Comme le démontre Panaccio dans son étude, Guillaume d'Ockham est celui qui en a présenté la version la plus complète en ce qui a trait aux périodes de l'Antiquité et du Moyen Âge. L'influence immédiate de ce penseur du quatorzième siècle à l'égard du langage intérieur fut très importante, mais s'est diluée par la suite. L'idée n'a pas disparu pour autant et a repris de la vigueur ces dernières décennies. Malgré tout, les cognitivistes contemporains qui défendent la thèse du language of thought méconnaissent, dans presque tous les cas, les développements du passé sur l'oratio mentalis, de même que les médiévistes ne s'intéressent guère de nos jours à cette problématique actuelle. La grande force de l'ouvrage que nous présente Panaccio est justement celle de perpétuer le dialogue qui constitue, finalement, le cœur même de l'entreprise philosophique. L'étude permet ainsi que se manifeste une curiosité réciproque entre des domaines philosophiques qui s'ignorent le plus clair du temps. Et pour nous, médiévistes, il s'agit là d'une manière concrète et efficace de souligner à nos contemporains que notre objet d'étude n'a pas qu'un intérêt historique, mais qu'il peut également servir à résoudre certains problèmes d'ordres pratique et théorique. Bien qu'il s'agisse d'un ouvrage dont les nombreuses problématiques abordées sont souvent complexes et dont certaines donnent parfois le vertige en raison de la profusion impressionnante d'informations données sur tel ou tel aspect du discours intérieur, comme le passage sur "Le jeu des triades" dans lequel sont présentées de multiples classifications ternaires des différents sens du mot verbum - l'A. se réfère lui-même à ces triades en utilisant l'expression révélatrice de "tourbillon de triades" (p. 167) —, on peut conclure que Panaccio a, au bout du compte, remporté son enjeu initial qu'il énonce ainsi dans son introduction : "Le pari de ce livre est que cette interrogation (de savoir où, comment et pourquoi s'est développée, de Platon à Guillaume d'Ockham, l'idée d'une pensée abstraite et discursive, indépendante des langues mais constituée de signes et dotée comme elles d'une syntaxe et d'une sémantique compositionnelles finement articulées) mette au jour une histoire doctrinale, riche et philosophiquement intéressante » (p. 26).

GUY HAMELIN

Universidade de Brasília

Jonas, Hans, Le phénomène de la vie : vers une biologie philosophique, trad. D. Lories, De Boeck Université, Bruxelles, 2001, 288 p. (Coll. Sciences, éthiques, sociétés)

Le Phénomène de la vie est un recueil d'études rassemblées par Hans Jonas ; il propose une philosophie de la vie susceptible d'éclairer et de fonder son éthique de la responsabilité. En effet, outre les recherches de Jonas sur le gnosticisme, le public connaissait Le Principe Responsabilité, traduit par Jean Greisch en 1990, qui propose une éthique prenant en compte le risque majeur que fait courir à l'homme la civilisa- 